\title{
ANKLE MUSCLE SYNERGIES FOR SMOOTH PEDAL OPERATION UNDER VARIOUS LOWER-LIMB POSTURE
}

\author{
Kazuo Kiguchi ${ }^{1}$, Takuto Fujita ${ }^{1}$, Sho Yabunaka $^{2}$, Yusaku Takeda ${ }^{2}$, \\ and Toshihiro Hara ${ }^{2}$ \\ ${ }^{1}$ Department of Mechanical Engineering, Kyushu University, Fukuoka, Japan \\ ${ }^{2}$ Mazda Motor Corporation, Fuchu-cho, Hiroshima, Japan
}

\begin{abstract}
A study on muscle synergy of ankle joint motion is important since the acceleration operation results in automobile acceleration. It is necessary to understanding the characteristics of ankle muscle synergies to define the appropriate specification of pedals, especially for the accelerator pedal. Although the biarticular muscle (i.e., gastrocnemius) plays an important role for the ankle joint motion, it is not well understood yet. In this paper, the effect of knee joint angle and the role of biarticular muscle for pedal operation are investigated. Experiments of the pedal operation were performed to evaluate the muscle synergies for the ankle plantar flexion motion (i.e., the pedal operation motion) in the driving position. The experimental results suggest that the muscle activity level of gastrocnemius varies with respect the knee joint angle, and smooth pedal operation is realized by the appropriate muscle synergies.
\end{abstract}

\section{KEYWORDS}

Ankle Joint, Pedal Operation, EMG, BiArticular Muscle, Muscle Synergy

\section{INTRODUCTION}

In order to design an automobile, human characteristics such as human musculoskeletal characteristics must be taken into account. Control of ankle joint motion is important for many tasks such as operation of an automobile. In the case of automobile operation, acceleration of the automobile is controlled with an acceleration pedal using the driver's ankle joint motion. Therefore, it is important to understand the characteristics of ankle muscle synergies to define the appropriate specification of pedals, especially for the accelerator pedal since its operation directly results in the acceleration of the automobile. Although the ankle joint motion is generated with several muscles, it is known that the biarticular muscle such as the gastrocnemius plays an important role for the joint motion [1]-[4]. Since gastrocnemius is a biarticular muscle, not only the ankle joint angle, but also the knee joint angle affects the muscle activity of gastrocnemius [4]. Therefore, lower-limb posture must be taken into account to consider the role of gastrocnemius muscle activity for the ankle joint motion, especially for plantar flexion motion. Consequently, the driving position affects the performance of muscle synergy of the ankle joint. However, the role of biarticular muscles is still not well understood [5] although the role of gastrocnemius activity for the ankle joint motion must be considered to understand ankle muscle synergies, especially for accelerator pedal operation.

Dhinaharan Nagamalai et al. (Eds) : CoSIT, SIGL, AIAPP, CYBI, CRIS, SEC, DMA - 2017

pp. 71- 78, 2017. (C) CS \& IT-CSCP 2017

DOI : $10.5121 /$ csit.2017.70408 
In this paper, the effect of knee joint angle and the role of biarticular muscle for pedal operation are investigated. Experiments of the pedal operation were performed to evaluate the muscle synergies for the ankle plantar flexion motion (i.e., the pedal operation motion) in the driving position. Electromyographic (EMG) activities of the certain important muscles were measured under several lower-limb postures (i.e., under several driving position) considering the pedal operation of an automobile in the experiment. The experimental results suggest that the muscle activity level of gastrocnemius varies with respect the knee joint angle, and smooth pedal operation is realized by the appropriate muscle synergies. This study is also important to control robotic devices [6] to assist the ankle joint motion according to the user's motion intention since EMG activities reflect the user's motion intention [7].

\section{EMG ACTIVITIES FOR PedAl OPERATION}

Experiments were performed to measure EMG activities of the certain important muscles of the ankle joint during the pedal operation motion in the driving position. In the experiment, the human subjects sat on the driving seat with the several different driving positions and then performed the pedal operation to investigate the effect of lower-limb posture. The relation between the accelerator pedal operation motion and the EMG activities of the certain muscles of the ankle joint was measured in the experiment.

\subsection{Experimental Setup}

The experimental setup is shown in Fig. 1. It mainly consists of a driving seat, an accelerator pedal, and a display. The relative position between the driving seat and the accelerator pedal can be changed. The ankle joint angle is measured with a goniometer. In the experiment, an ordinal accelerator pedal is used and the scenery on the display is changed in accordance with the pedal operation by the driver.

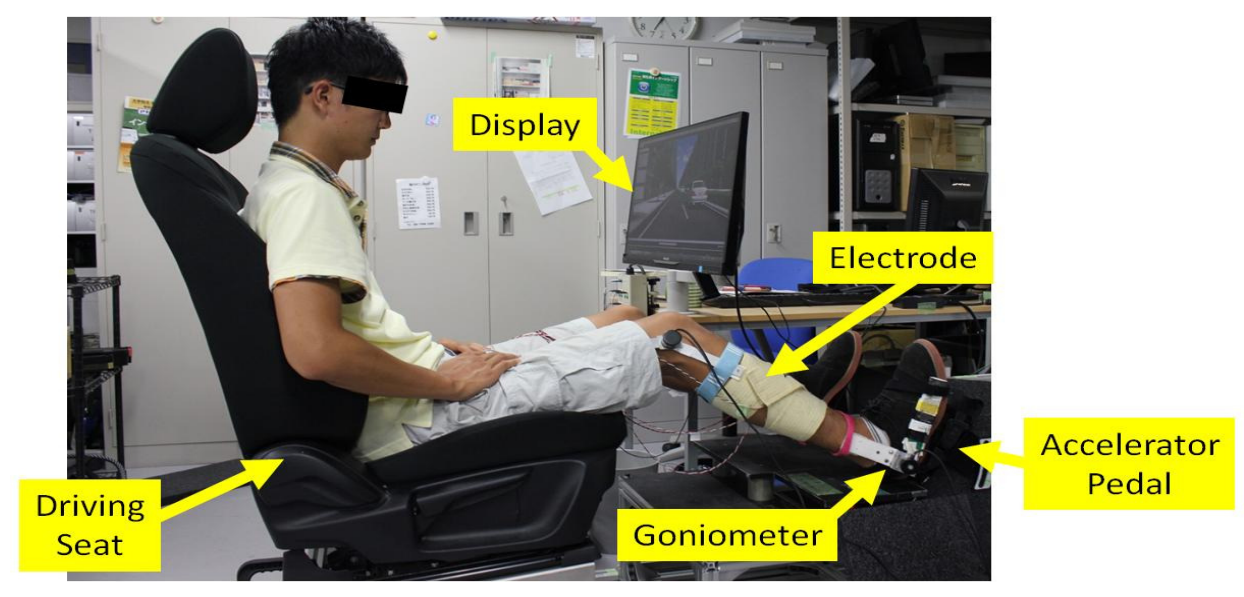

Figure 1. Experimental setup

\subsection{Measurement}

The EMG signals of the important muscles for the ankle joint motion (i.e., tibialis anterior, peroneus longus, gastrocnemius, and soleus) are measured in the experiment. The location of each electrode for EMG measurement is shown in Fig. 2. The EMG signals from the electrodes are amplified and sent to the computer. In order to extract a feature of the EMG signal, the Root Mean Square (RMS) is calculated as shown below. 


$$
R M S=\sqrt{\frac{1}{N} \sum_{i=1}^{N} v_{i}^{2}}
$$

where $N$ is the number of samples in a segment, $v_{i}$ is the voltage at $i^{\text {th }}$ sampling point. The number of samples $N$ is set to be 100 and the sampling frequency is $500 \mathrm{~Hz}$ in this study. Then it is transferred to \% MVC.
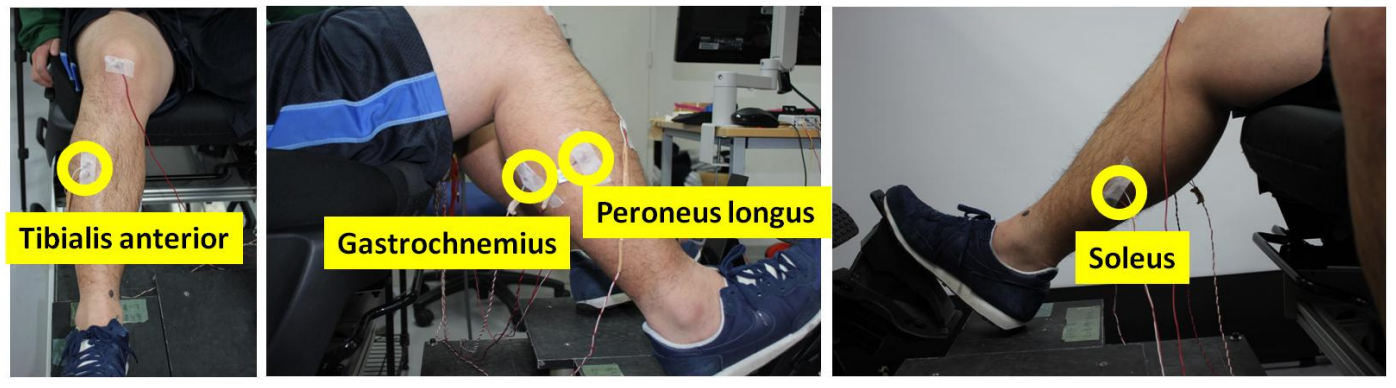

Figure 2. Location of each electrode

\subsection{Experiment of Pedal Operation}

Experiments were carried out with three human subjects. Basically, three types of seat position (sedan, SUV, and sport types) are prepared for the experiments considering the types of automobile as shown in Fig. 3. The angle of the acceleration pedal is also different in each seat type. Another three kinds of seat slide position (close, appropriate, and far positions) are prepared in the experiments considering the driving position in an automobile. Examples of these positions in the sedan type are shown in Fig. 4. The details of the initial lower-limb posture are written in Table 1. Here, the knee angle means the knee joint flexion angle from the extended knee position and the ankle angle means the ankle joint plantar flexion angle with respect to the shank axis.

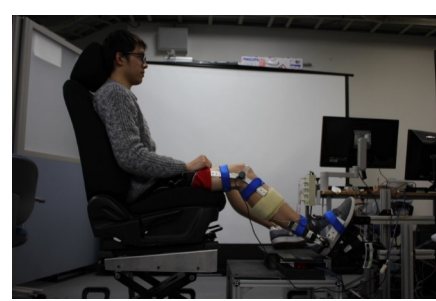

(a) Sedan type

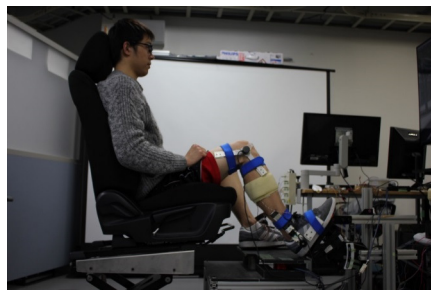

(a) Close position

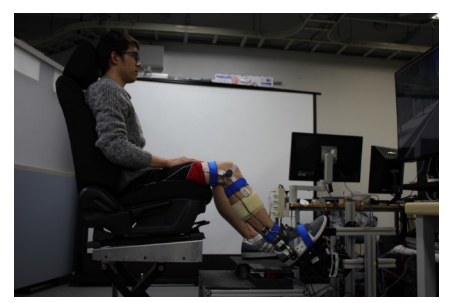

(b) SUV type

Figure 3. Seat Positions

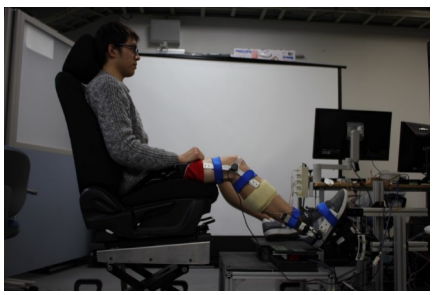

(b) Appropriate position

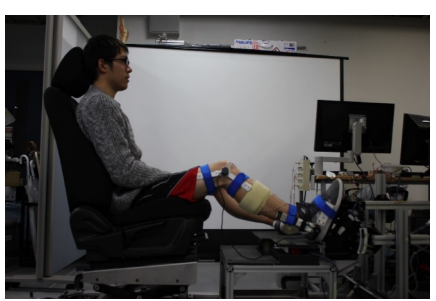

(c) Sport type

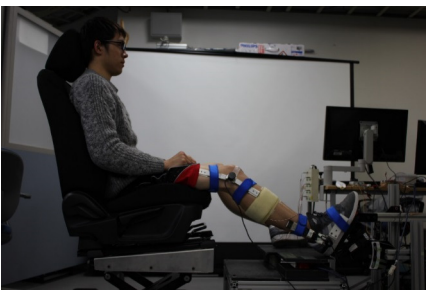

(c) Far position

Figure 4. Seat Slide Positions (Driving Positions) in Sedan Type 
In the experiment, another automobile is running at $60[\mathrm{~km} / \mathrm{h}]$ to the same direction in the next lane on the display. The subject (driver) operates the accelerator pedal to accelerate the automobile from the state of rest to catch up with another automobile in the next lane. Then, the subject operates the accelerator pedal to make the automobile run side by side in the first experiment.

In the second experiment, the subject (driver) catches up with another automobile which is running at $60[\mathrm{~km} / \mathrm{h}]$ in the next lane and makes the automobile run side by side for a while, then overtakes another automobile in the next lane after a while.

Table 1. Initial Lower-Limb Postures.

\begin{tabular}{|c|c|c|c|c|}
\hline Subject (Height) & Seat Type & Seat Position & Knee Angle [deg] & Ankle Angle [deg] \\
\hline \multirow{9}{*}{ A $(1.79 \mathrm{~m})$} & \multirow{3}{*}{ Sedan } & Close & 91.7 & 68.4 \\
\hline & & Appropriate & 137.5 & 80.8 \\
\hline & & Far & 149.6 & 87.8 \\
\hline & \multirow{3}{*}{ SUV } & Close & 99.2 & 61.3 \\
\hline & & Appropriate & 133.8 & 82.9 \\
\hline & & Far & 146.5 & 87.6 \\
\hline & \multirow{3}{*}{ Sport } & Close & 100.9 & 76.6 \\
\hline & & Appropriate & 146.9 & 90.9 \\
\hline & & Far & 155.9 & 93.7 \\
\hline \multirow{9}{*}{ B $(1.66 \mathrm{~m})$} & \multirow{3}{*}{ Sedan } & Close & 98.7 & 70.8 \\
\hline & & Appropriate & 127.6 & 85.2 \\
\hline & & Far & 145.3 & 89.9 \\
\hline & \multirow{3}{*}{ SUV } & Close & 99.2 & 61.3 \\
\hline & & Appropriate & 122.7 & 82.9 \\
\hline & & Far & 140.1 & 87.6 \\
\hline & \multirow{3}{*}{ Sport } & Close & 109.6 & 80.2 \\
\hline & & Appropriate & 131.4 & 96.1 \\
\hline & & Far & 149.7 & 113.4 \\
\hline \multirow{9}{*}{$\mathrm{C}(1.78 \mathrm{~m})$} & \multirow{3}{*}{ Sedan } & Close & 100.6 & 74.1 \\
\hline & & Appropriate & 120.2 & 86.8 \\
\hline & & Far & 150.7 & 96.9 \\
\hline & \multirow{3}{*}{ SUV } & Close & 98.5 & 66.1 \\
\hline & & Appropriate & 119.4 & 82.8 \\
\hline & & Far & 137.5 & 85.9 \\
\hline & \multirow{3}{*}{ Sport } & Close & 100.9 & 78.2 \\
\hline & & Appropriate & 115.4 & 92.6 \\
\hline & & Far & 159.7 & 100.5 \\
\hline
\end{tabular}

\section{EXPERIMENTAL RESULTS}

Figures 5-7 show the results of the first experimental of the subject A with the sedan, SUV, and sport type seat positions, respectively. These results show that the activities of muscles for plantar flexion such as peroneus longus, soleus, and gastrocnemius increase as the seat slide position becomes further from the acceleration pedal since the knee joint is extended and the ankle dorsiflexion angle is decreased. Especially, the increase the ratio of monoarticular muscle peroneus lingus activity is prominent. The ratio of biarticular muscle gastrocnemius varies depends on the seat position. These results show that the muscle synergy of the ankle joint motion for the same acceleration pedal operation varies in accordance with the knee angle and the ankle angle of the driver. 


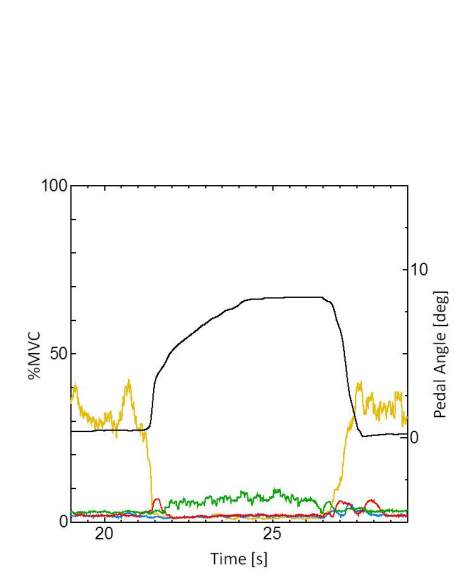

(a) Close position

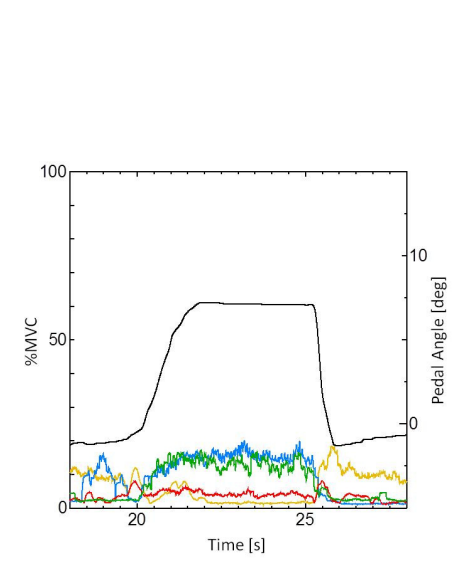

(b) Appropriate position

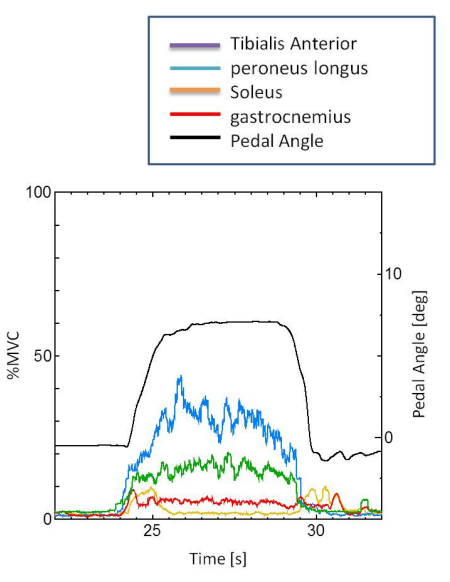

(c) Far position

Figure 5. Experimental Results in Sedan Type (Subject A)

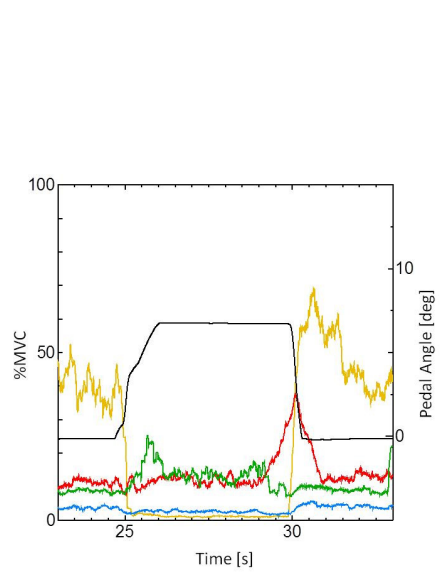

(a) Close position

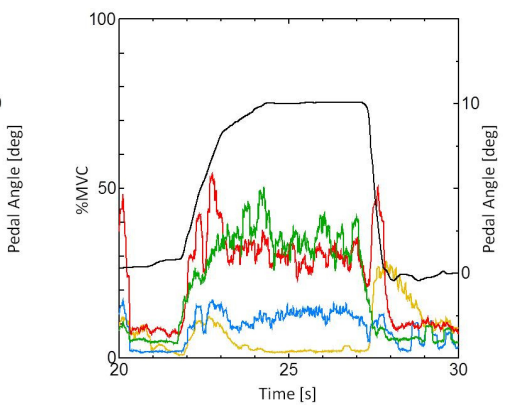

(b) Appropriate position

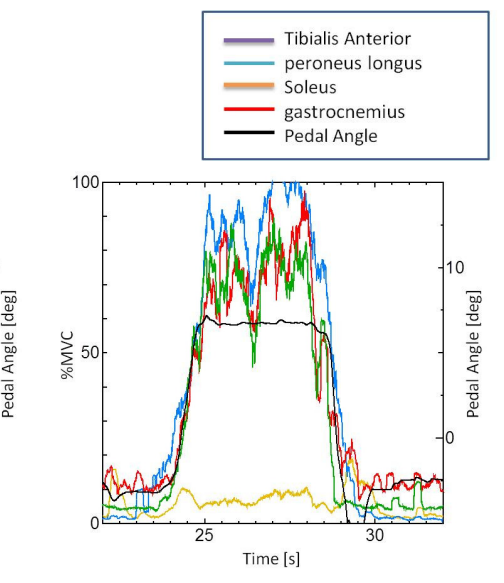

(c) Far position

Figure 6. Experimental Results in SUV Type (Subject A)

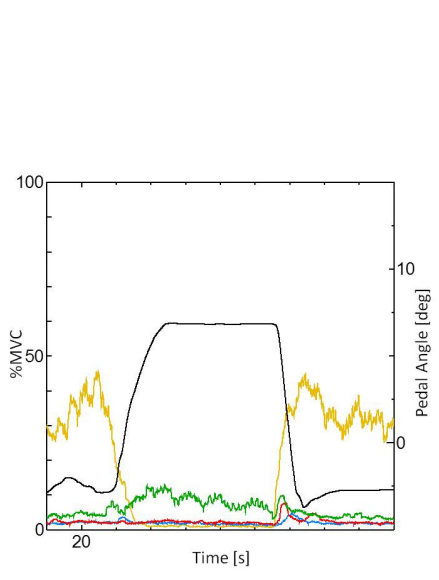

(a) Close position

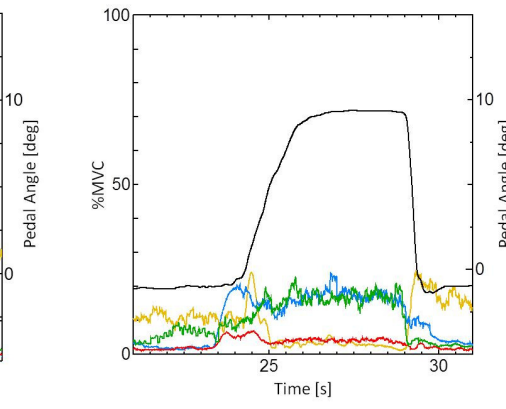

(b) Appropriate position

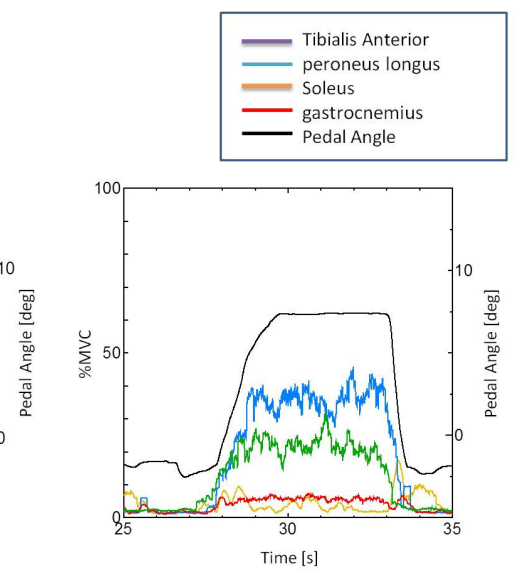

(c) Far position

Figure 7. Experimental Results in Sport Type (Subject A) 
The angle of the acceleration pedal also affects the muscle synergy of the ankle joint. Even though the initial lower-limb posture in the far seat position in the SUV seat type and that in the appropriate seat position in the sport seat type of the subject A is almost the same, the muscle activity level is different since the angle of the acceleration pedal is different. The same tendency can be observed with the experimental results with the other subjects.

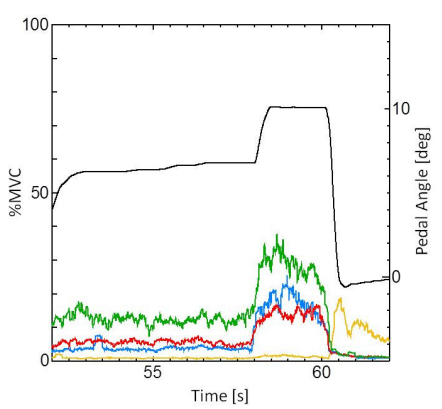

(a) Sedan type

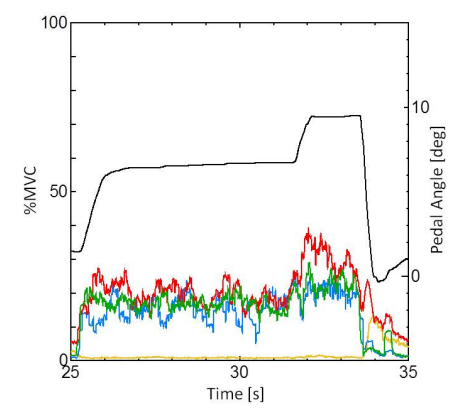

(b) SUV type

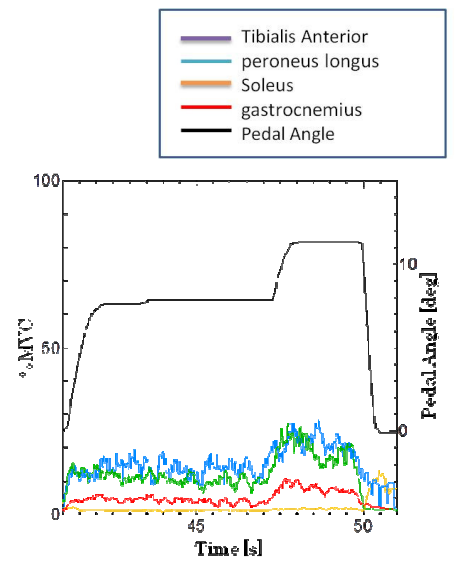

(c) Sport type

Figure 8. Experimental Results of Overtaking (Subject A)

The results of the second experimental of the subject A in the appropriate seat position with the sedan, SUV, and sport type seat are shown in Fig. 8. The results show that the activity levels of muscles for plantar flexion such as peroneus longus, soleus, and gastrocnemius increase when the angle of the acceleration pedal is increased for the overtaking. Note that the ratio of each muscle for the plantar flexion varies when the angle of the acceleration pedal is increased for the overtaking.

\section{DISCUSSION}

The experimental results show that the activity levels of muscles for plantar flexion such as peroneus longus, soleus, and gastrocnemius increase as the seat slide position becomes further from the acceleration pedal since the knee joint is extended and the ankle dorsiflexion angle is decreased. As one can see in Table 1, the most of the initial ankle joint angles are slightly dorsiflexed angles, especially in the case of the close seat position. Therefore, the muscle for the dorsiflexion (i.e., tibialis anterior) is released to make the plantar flexion motion. In this case, the plantar flexion motion can be generated with a little activity of the muscles for plantar flexion. Since the gastrocnemius is a biarticular muscle, the knee joint angle affects the activity level. Arampatzis et. al [4] also suggest that a critical force-length potential of muscles of the triceps surae results in the decrease of EMG activity of the gastrocnemius medialis at pronounced knee flexed positions.

As the seat slide position becomes further from the acceleration pedal, the initial ankle joint angles become closer to the plantar flexed angles. Therefore, further plantar flexion motion is necessary to operate the acceleration pedal. Consequently, the muscle activity levels of the muscles for the plantar flexion become higher. The experimental results shown in Figs. 5-7 suggest that the ratio of each muscle for the plantar flexion effectively varies in accordance with the lower-limb posture and the angle of the accelerator pedal to make the smooth pedal operation. The experimental results shown in Fig. 8 suggest that the ratio of each muscle for the plantar flexion moderately varies when the angle of the acceleration pedal is increased for the overtaking 
since the reaction force from the pedal is increased. When the angle of the acceleration pedal is increased, the activity level of the gastrocnemius becomes a little higher instantly sometimes. Ingen Schenau et. al [1] showed that the biarticular muscles such as the gastrocnemius are used to transport energy from proximal to distal joints during jumping. Therefore, the change of the muscle synergy might be concerned with the energy transmission. The experimental results suggest that the muscle synergy of the ankle plantar flexion motion for the acceleration pedal operation is moderately controlled according to the condition. Further study is required to understand the muscle synergy of ankle joint motion for acceleration pedal operation

\section{CONCLUSIONS}

In this paper, the effect of knee joint angle and the role of biarticular muscle for ankle joint motion (i.e., pedal operation) were investigated. The experimental results showed that muscle synergy of ankle joint motion for acceleration pedal operation moderately varies in accordance with the condition such as the lower-limb posture of the driver, the angle of the acceleration pedal, and the amount of the required ankle torque.

\section{REFERENCES}

[1] Ingen Schenau, G.J. van, Bobbert, M.F., \& Rozendal, R.H., (1987) "The unique action of bi-articular muscles in complex movements", Journal of Anatomy, vol. 155, pp1-5.

[2] Kumamoto, M., Oshima, T., \& Yamamoto, T., (1994) "Control properties induced by the existence of antagonistic pairs of bi-articular muscles - Mechanical engineering model analyses”, Human Movement Science, vol. 13, pp611-634.

[3] Lee, S.S.M. \& Piazza, S.J., (2008) "Inversion-eversion moment arms of gastrocnemius and tibialis anterior measured in vivo", Journal of Biomechanics, vol. 41, pp3366-3370.

[4] Arampatzis, A., Karamanidis, K., Stafilidis, S., Morey-Klapsing, G., DeMonte, G., \& Bruggemann, G.P., (2006) "Effect of different ankle- and knee-joint positions on gastrocnemius medialis fascicle length and EMG activity during isometric plantar flexion”, Journal of Biomechanics, vol. 39, pp18911902.

[5] Cleather, D.J., Southgate, D.F.L., Stafilidis, S., \& Bull, A.M.J., (2015) "The role of the biarticular hamstrings and gastrocnemius muscles in closed chain lower limb extension", Journal of Theoretical Biology, vol. 365, pp217-225.

[6] Jimenez-Fabian, R. \& Verlinden, O., (2012) "Review of control algorithms for robotic ankle systems in lower-limb orthoses, prostheses, and exoskeletons”, Medical Engineering \& Physics, vol. 34, pp397-408.

[7] Kiguchi, K. \& Hayashi, O., (2012) "An EMG-Based Control for an Upper-Limb Power-Assist Exoskeleton Robot”, IEEE Trans. on Systems, Man, and Cybernetics, Part B, Vol. 42, no. 4, pp10641071

\section{AUTHORS}

Kazuo Kiguchi received the B.E. degree in mechanical eng. from Niigata Univ., Japan in 1986, the M.A.Sc. degree in mechanical eng. from the Univ. of Ottawa, Canada in 1993, and the Doctor of .Eng. degree in Mechano-Informatics from Nagoya Univ., Japan in 1997. He is currently a professor in the Dept. of Mechanical Engineering, Faculty of Eng., Kyushu University, Japan. His research interests include biorobotics, human assist robots, and health care robots.

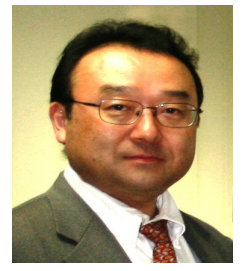


Takuto Fujita is currently a student in mechanical and aerospace eng., Faculty of Eng., Kyushu Univ., Japan

Sho Yabunaka received received the BSc. degree in Culture and Information Science from Doshisha University Japan, in 2010, and MBIT. degree in Information Science from Nara Institute of Science and Technology, Japan, in 2012. He is currently a Specialist of Kansei Engineering Research, Advanced Human-Vehicle Research Field, Technical Research Center, Mazda Motor Corporation. His current research interests include the mechanism of human musculoskeletal system and these applications for designing vehicle.

Yusaku Takeda received the B.E. and M.E degrees in mechanical systems and design eng. from Hiroshima Univ., Japan, in 2000 and 2003, and Doctor of Engineering degree in Artificial Complex Systems Eng. from Hiroshima Univ. in 2005. He is currently a Senior Specialist of Kansei Engineering Research, Advanced Human-Vehicle Research Field, Technical Research Center, Mazda Motor Corporation. His current research interests include the mechanism of human musculoskeletal system and visual awareness, and these applications for designing vehicle.

Toshihiro Hara received the B.E. degrees in electrical engineering from Himeji Institute of Technology, Japan, in 1991. He is currently a Technical Leader of Kansei Engineering Research, Advanced Human-Vehicle Research Field, Technical Research Center, Mazda Motor Corporation. His current research interests include the novel HMI design method based on the ergonomics, and these applications for designing vehicle.

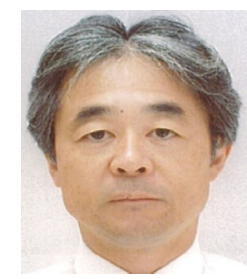

